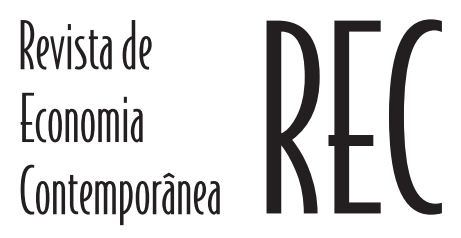

Revista de Economia Contemporânea (2016) 20(1): p. 177-200

(Journal of Contemporary Economics)

ISSN 1980-5527

http://dx.doi.org/10.1590/198055272017

www.ie.ufrj.br/revista

www.scielo.br/rec

\title{
A POBREZA MULTIDIMENSIONAL NO ESTADO DA BAHIA DIMINUIU? EVIDÊNCIAS A PARTIR DA ABORDAGEM DE BOURGUIGNON E CHAKRAVARTY
}

\author{
Áydano Ribeiro Leite ${ }^{a}$ \\ Andréa Ferreira da Silva ${ }^{b}$ \\ Jair Andrade de Araújo ${ }^{c}$ \\ Geidson Uilson Seixas Santana ${ }^{d}$ \\ aDoutorando em Economia aplicada pela Universidade Federal da Bahia (UFBA) e professor assistente da \\ Universidade Regional do Cariri (URCA). \\ ${ }^{\text {b}}$ Doutoranda em Economia aplicada pela Universidade Federal da Paraíba (UFPB). \\ 'Professor adjunto da Universidade Federal do Ceará (UFC). \\ ${ }^{\mathrm{d}}$ Doutorando em Economia Aplicada pela UFBA.
}

Artigo recebido em 16/06/2015 e aprovado em 05/02/2016.

RESUMO: A maioria das análises sobre o fenômeno da pobreza tem dado um enfoque especial a sua estrutura unidimensional, em que se considera a insuficiência de renda dos indivíduos. Destarte, mais recentemente muitos pesquisadores têm abordado a pobreza do ponto de vista multidimensional, considerando outros aspectos além da renda. Nesse sentido, este artigo tem por objetivo investigar a pobreza multidimensional no estado da Bahia no período compreendido entre 2006 e 2013. Para tanto, utilizou-se a recente metodologia proposta por Bourguignon e Chakravarty (2003), considerando a abordagem das necessidades básicas e a teoria das capacitações. Assim, para o cálculo dos indicadores de privação por dimensão, seus gaps e os respectivos índices de pobreza multidimensional, foram utilizados dados da Pesquisa

Correspondência para: Áydano Ribeiro Leite.

Contato: economistaaydano@yahoo.com.br. 
Nacional por Amostragem de Domiciliar (PNAD). Os resultados apontam uma retração de 4,41\% da pobreza multidimensional no estado da Bahia, enquanto nas áreas rural e urbana, a proporção de pobres diminuiu 5,61\% e 4,39\% respectivamente. Por outro lado, quando se trata dos grupos que compõem a análise, a redução da pobreza para os gêneros masculino e feminino foi relativamente equitativa. Entretanto, pode-se destacar significativa retração do indicador relacionado às crianças em 5,47\% no período analisado.

PALAVRAS-CHAVE: pobreza multidimensional; privação; estado da Bahia.

CLASSIFICAÇÃO JEL: I32; $\mathrm{CO}^{2}$. 


\title{
HAS MULTIDIMENSIONAL POVERTY IN THE STATE OF BAHIA DECREASED? EVIDENCES BASED ON BOURGUIGNON AND CHAKRAVARTY'S APPROACH
}

\begin{abstract}
Most analysis of the poverty phenomenon have given special focus to their one-dimensional structure, which is defined as insufficient income of individuals. However, more recently many researchers have addressed the multidimensional poverty perspective, taking into consideration other aspects in addition to income. With that respect, this article aims to investigate the multidimensional poverty in the state of Bahia in the period between 2006 and 2013. To this end, a recent methodology proposed by Bourguignon and Chakravarty (2003) has been employed, based on the basic needs approach and the theory of training. For the sake of calculating the size deprivation indicators, related gaps and multidimensional poverty rates, we used data from the National Survey by Household Sampling (PNAD/IBGE) .The results show that multidimensional poverty has decrease by $4.41 \%$ in the state of Bahia, while in rural and urban areas, the proportion of poor declined by $5.61 \%$ and $4.39 \%$ respectively. On the other hand, when it comes to the groups that make up the analysis, poverty reduction for males and females was relatively fair. However, it can highlight significant retraction of the children related indicator in $5.47 \%$ in the period analyzed.
\end{abstract}

KEYWORDS: multidimensional poverty; privation; rural area; state of Bahia; Brazil.

\section{INTRODUÇÃO}

Nos últimos anos o debate sobre os problemas sociais no Brasil ligados à pobreza, suas causas e consequências tornaram-se centrais no âmbito acadêmico. Nesse aspecto, as políticas de combate ao problema têm focado os níveis de pobreza correlacionando-os, sobretudo, às restrições monetárias ou unidimensionais sem levar em consideração a pobreza em suas múltiplas dimensões. Para Silva e Barros (2006), dois fatores explicam essa tendência. O primeiro estaria relacionado à ideia da natureza escalar das medidas de pobreza pautadas na insuficiência de renda. Um segundo aspecto importante é a relação existente entre renda e bem-estar, visto que a insuficiência de renda dos indivíduos restringe o acesso ao mercado de bens. 
Destarte, destacam-se como formas de observação da pobreza as vertentes unidimensional e multidimensional. A primeira relaciona-se fortemente à ideia de insuficiência de renda necessária à manutenção física do indivíduo. A segunda enfatiza a complexidade do fenômeno, destacando que a pobreza não se limita aos elementos materiais e individuais. Dessa forma, a pobreza multidimensional implicitamente releva questões mais amplas, como os problemas de saúde, educação, o baixo padrão de vida, a insuficiência de renda, a baixa qualidade do trabalho e a suscetibilidade à violência. Portanto, a perspectiva multidimensional apresenta elementos mais consistentes com a realidade dos indivíduos, no que tange à mensuração da pobreza, do que a vertente unidimensional.

Do ponto de vista metodológico, o indicador proposto por Anand e Sen (1997) ainda permanece como principal referência de estratégia empírica na investigação da pobreza multidimensional. Contudo, nesta pesquisa utilizou-se a metodologia proposta por Bourguignon e Chakravarty (2003) a partir de uma medida que explicite a pobreza e sua intensidade a depender dos diversos indicadores de renda e não rendimento. Por outro lado, espera-se que as evidências empíricas aqui apontadas a partir de tal método possam subsidiar a formulação de políticas públicas em direção à redução dos níveis de pobreza, sobretudo, nas áreas rurais.

Nesse sentido, o objetivo central deste trabalho foi identificar e analisar a evolução e comportamento dos principais indicadores de pobreza multidimensional no estado da Bahia, levando em consideração os espaços geográficos, grupos de gênero e raça, além dos rebatimentos da pobreza sobre faixas de idade. Portanto, considerando a relevância dos programas de transferência de renda e os avanços sobre a escolaridade dos indivíduos ao longo do período e seus possíveis efeitos sobre os principais indicadores sociais, será que a pobreza multidimensional reduziu-se de forma significativa no estado da Bahia?

Além da introdução e conclusão, este trabalho está dividido em mais três seções. Na seção 1 serão apresentadas as principais discussões dentro da literatura especializada enfocando a pobreza multidimensional em seus vários aspectos. Na seção 2 serão discutidas a metodologia e a estratégia empírica adotadas na pesquisa proposta por Bourguignon e Chakravarty. Por fim, na seção 3 serão expostos os resultados divididos em análise de incidência de privação de cada dimensão e seus correspondentes gap, além dos índices de pobreza multidimensional. 


\section{REVISÃO DA LITERATURA}

\subsection{PRINCIPAIS ABORDAGENS TEÓRICAS}

Conforme Alkire e Foster (2011), a perspectiva unidimensional baseada na insuficiência de renda e disseminada desde a década de 1970 assumiu protagonismo diante das investigações acerca dos níveis de pobreza. No entanto, estudos recentes ganharam força na assertiva de considerar a pobreza um fenômeno multidimensional, o que levou alguns autores a buscarem formas alternativas de analisá-la. De acordo com Salama e Destremau (1999), a ideia era a de incorporar à pobreza e a seus meios de medidas dimensões não monetárias e particularmente sociais e políticas. Nesse contexto, no arcabouço multidimensional destacam-se duas abordagens: a das necessidades básicas e a abordagem das capacitações.

Na perspectiva das necessidades básicas, algumas variáveis são consenso dentre os especialistas. Nesse sentido, destaca-se o acesso à água potável, à rede de esgoto, à coleta de lixo, ao transporte público e à educação como bens imprescindíveis para os indivíduos usufruírem de uma vida saudável e com chances de inserção social. Por sua vez, Rocha (2006) incorpora uma noção mais ampla das necessidades humanas, por entender que a de pobreza abrange outros aspectos da vida cotidiana dos indivíduos. Assim, é importante considerar o fato de que os indivíduos não apenas se alimentam, mas se relacionam, trabalham, enfim, têm uma vida social. Portanto, a abordagem das necessidades básicas ultrapassa as definições concernentes às questões alimentares ou nutricionais, incorporando elementos como lazer, transporte, educação, saneamento, habitação entre outros.

Quanto à abordagem das capacidades, Thorbecke (2008) admite que o ponto de inflexão na formulação dessa teoria é o programa de pesquisa desenvolvido pelo economista Amartya Sen. Portanto, a partir de então, acresce ao debate o suposto de que a visão da pobreza como privação de renda é limitada e pode ser danosa ao seu enfrentamento, por ofuscar as perspectivas mais complexas e delicadas da condição humana. Além do avanço conceitual, atualmente observa-se um conjunto de ferramentas empíricas que identificam e localizam os pobres, além de descrever suas características e medir o grau de pobreza em diferentes níveis de agregação.

Conforme Kuklys (2005), a abordagem das capacidades opera claramente em dois níveis. O primeiro refere-se à realização de bem-estar, que é mensurado em termos de "funcionamentos" que refletem os vários acontecimentos ou bens que um indivíduo pode considerar valioso fazer ou ter. $\mathrm{O}$ segundo se refere ao potencial de bem-estar que é estimado em termos de "capacidades", em que um indivíduo consiste nas diversas combinações de funcionamentos cuja realização é possível para ele. 
Bourguignon e Chakravarty (2003) estruturam uma medida que capte consistentemente a pobreza e sua intensidade a depender de indicadores de renda e de não rendimento. Tais indicadores podem ajudar na identificação de aspectos de bem-estar não capturado pela renda. Portanto, há inadequação na utilização da renda como único indicador de bem-estar. Dessa maneira, para incorrer em medidas mais completas, outros atributos ou variáveis devem compor o processo de construção dos indicadores (moradia, alfabetização, expectativa de vida, provisão de bens públicos etc.). $\mathrm{O}$ bem-estar é intrinsecamente multidimensional sobre o ponto de vista das capacitações e funcionamentos. Com efeito, funcionamentos são minuciosamente motivados por atributos como capacidade de ler e escrever, expectativa de vida, entre outros, e não apenas pela renda.

Dentro desse aspecto, a renda per capita, portanto, limita a compreensão de fatores subjacentes ao bem-estar. Dessa maneira, quando se toma o indicador de renda como único mecanismo de verificação da prosperidade de uma nação, inviabiliza-se a análise dos demais indicadores de bem-estar da população, pois um país com alta renda per capita, mas com acesso restrito aos serviços de saúde e educação básica, pode ter menor expectativa de vida e maiores taxas de mortalidade (SEN, 1993).

Segundo UNPD (1997), a ausência de oportunidades para se viver uma vida suportável aponta para a necessidade do aspecto multidimensional para observação do fenômeno da pobreza. Entretanto, há criticas que salientam que a estimativa empírica da pobreza multidimensional é dificultada pela necessidade de ponderação de múltiplos conceitos de pobreza em um único indicador. Além disso, do ponto de vista qualitativo, a abordagem multidimensional da pobreza pode criar um conflito entre as suas causas e a real pobreza, referente à renda, o que pode levar a uma subestimação do papel da renda sobre esse fenômeno.

Cabe destacar que a opção pelo enfoque da pobreza multidimensional não significa o abandono da renda como uma dimensão da pobreza, embora alguns trabalhos não incluam essa variável. Sen (2000) observa que o comum é a inclusão da insuficiência monetária e indicadores não monetários. Portanto, destaca-se que a renda é uma importante dimensão da pobreza, mas não pode ser considerada a única. Dessa forma, a pobreza baseada na privação da renda é de grande valia na compreensão do fenômeno, já que a insuficiência de renda é limitadora dos atos dos indivíduos e a principal causa da fome individual e coletiva.

Destarte, os níveis de renda são relevantes, pois permitem que as pessoas adquiram bens e serviços e que usufruam de um determinado padrão de vida. Dessa forma, verifica-se a presença da dimensão renda na maioria dos estudos multidimensionais. Essa opção é incluída em diversos estudos com a abordagem das necessidades básicas e das capacidades. Nesse sentido, o enfoque nesse estudo abarca o método que trata a pobreza pela lógica multidimensional, associando a renda a diversos indicadores, referenciados na literatura nacional e internacional sobre o tema. 


\subsection{A DETERMINAÇÃO DA POBREZA MULTIDIMENSIONAL: INDICADORES E DIMENSÕES}

De acordo com Sen (1976), a medição da pobreza multidimensional pode ser dividida conceitualmente em duas etapas distintas: identificação e agregação. A etapa da identificação pressupõe o estabelecimento de um parâmetro, chamado de linha de pobreza, que, quando confrontado a um indicador de bem-estar, permite a classificação da população em dois grupos: pobres e não pobres. Dessa forma identificam-se os pobres entre a população total, enquanto a agregação consiste na seleção de uma medida ou um índice de pobreza específico que agregue as informações sobre pessoas pobres em um indicador global de pobreza. Consiste, portanto, na construção de uma medida numérica da pobreza.

Bourguignon e Chakravarty (2003) ressaltam que, nas últimas décadas, os programas de pesquisas voltados ao entendimento e enfrentamento da pobreza avançaram significativamente. O debate no campo unidimensional e multidimensional tem possibilitado proposições importantes tanto de cunho monetário quanto não monetário para a elaboração de políticas públicas de enfrentamento ao fenômeno. A redução da pobreza ou a mitigação de suas consequências permanece, portanto, com um grande problema para a maioria dos países.

Mesmo com avanços recentes na literatura sobre a construção de indicadores escalares de pobreza multidimensional, ainda há relativa dominância dos estudos que utilizam o mecanismo unidimensional. Nesse sentido, a construção de indicadores escalares que abarquem múltiplas dimensões é de grande valia para a continuidade dos avanços alcançados.

Barros et al. (2006) frisam a importância de indicadores escalares de pobreza multidimensional, elencando alguns passos necessários à construção desse tipo de medidas. São eles: (1) selecionando dimensões e indicadores; (2) a opção por um escalar; (3) agregando indicadores e dimensões; (4) agregações e sequências das agregações; (5) agregando a pobreza dos agentes; (6) linhas de pobreza; e (7) medindo o grau de pobreza.

Há na literatura nacional e internacional métodos diversos de construção de indicadores multidimensionais da pobreza. A definição do método varia conforme a corrente, condições e acesso aos dados e pretensões do pesquisador. No Brasil, Barros et al. (2006) apontam alguns problemas metodológicos para o desenvolvimento do indicador multidimensional. Por exemplo, consideram que na comparação entre dois índices distintos, mesmo que construídos com dimensões ou variáveis idênticas, pode haver distinção entre os pesos de cada variável. Portanto, deve-se proceder com cautela na escolha do método e na construção dos indicadores e, sobretudo, nas análises que se tecem a partir desses indicadores. 
Destaca-se ainda que, em uma abordagem multidimensional, a análise é contextual. Assim, é importante acentuar que são equivocadas as comparações de dimensões de estudos diferentes entre países distintos. Portanto, a especificidade local gera informações relevantes para a tomada de decisões e políticas públicas pertinentes a cada região ou país. Nessa linha, o processo de seleção das dimensões deve considerar fortemente as características intrínsecas ao contexto que se pretende compreender, interpretar e ao qual se pretende propor políticas de correção e/ou mitigação da pobreza. Ainda no que tange à seleção das variáveis das dimensões, deve-se associar as especificidades locais com os "achados" na literatura especializada.

Segundo Mideros (2012), as variáveis podem ser identificadas tanto na abordagem das necessidades básicas quanto na abordagem das capacitações. São elas: educação, saúde, segurança alimentar, moradia, acesso aos serviços públicos básicos de infraestrutura e trabalho Além disso, o mecanismo precisa associar-se a metas de desenvolvimento factíveis com a realidade local.

\subsection{EVIDÊNCIAS SOBRE A POBREZA MULTIDIMENSIONAL NO ESTADO DO BAHIA}

De acordo com o IBGE (2010), o estado da Bahia tem uma população estimada em pouco mais de quinze milhões de habitantes. Esta população está distribuída em 417 municípios, dos quais $64 \%$ se encontram na região do semiárido. O rendimento nominal domiciliar per capita é de R\$697,00. No ano de 2014 estavam inscritos no CadÚnico $^{1}$ 9,4 milhões de baianos, dos quais 2,7 milhões de pessoas eram beneficiadas pelo Programa Bolsa Família. No geral, os indicadores sociais e econômicos da Bahia figuram entre os piores do Brasil, fruto de um processo histórico de desenvolvimento desigual engendrado por séculos. Ao longo das últimas décadas houve alguns avanços inegáveis no que tange à melhoria das condições de vida e acesso ao bem público, mas ainda há muito que se progredir, sobretudo no que concerne às necessidades básicas.

Alguns estudos foram realizados nos últimos anos para tentar captar a pobreza na Bahia para além da abordagem predominante, qual seja a da privação de renda. Assim, Lacerda e Neder (2010) desenvolveram um trabalho sobre pobreza multidimen-

\footnotetext{
Cadastro Único para Programas Sociais do Governo Federal é um instrumento que identifica e caracteriza as famílias de baixa renda, entendidas como aquelas que têm renda mensal de até meio salário mínimo por pessoa ou renda mensal total de até três salários mínimos. O Programa Bolsa Família atenderá às famílias em situação de pobreza e extrema pobreza, caracterizadas pela renda familiar mensal per capita de até $\mathrm{R} \$ 154,00$ (cento e cinquenta e quatro reais) e R \$77,00 (setenta e sete reais), respectivamente (Disponível em: www.mds.gov.br/bolsafamilia/cadastrounico).
} 
sional, definindo os anos de 1995, 2001 e 2006 como período de análise, utilizando a abordagem das Necessidades Humanas Básicas e das Capacidades como fundamentação teórica para desenvolver o indicador multidimensional de pobreza (IMP). Os autores destacam que o indicador multidimensional estabelecido para a Bahia não pode ser considerado como aquele que mede as capacidades, pois se trata apenas da interação entre as necessidades básicas e functionings (estados - o que se é), salientando, portanto, que o conceito das capacidades foi eleito como fundamento de análise. Além disso, ressalta-se que apesar do esforço, o trabalho contém limitações impostas pela fonte de dados utilizada e pela dificuldade de mensurar as diversas dimensões da pobreza.

Os principais resultados obtidos por Lacerda e Neder (2010), quando se analisa separadamente as regiões metropolitana, rural e urbana, apontam a área rural como aquela em que os níveis de pobreza são mais agudos. Esse resultado é observado nos três índices calculados para o estudo, descrevendo que a pobreza nessa área "era maior, mais intensa e mais severa”. No entanto, o estudo verificou que a pobreza rural, conforme metodologia utilizada, diminuiu no recorte temporal estabelecido. Por outro lado, houve crescimento da pobreza no período 1995-2006 na região metropolitana. No que tange à comparação do mecanismo multidimensional com o unidimensional (renda), a pobreza do ponto de vista multidimensional aflige um percentual maior do que o apontado pelo recorte de renda.

Outro trabalho que considera a abordagem multidimensional da pobreza é o da SEI (2014). O estudo utiliza o censo de 2010 para a análise de fatores além da insuficiência de renda. O objetivo foi observar, por meio da análise fatorial e de clusters, o comportamento da pobreza de forma espacial no estado da Bahia. Assim, delineou espaços denominados manchas, onde a pobreza se manifesta em seu caráter multidimensional. Além disso, a partir da proposta de clusters, foram identificados grupos de municípios com características aproximadas em relação às dimensões selecionadas. O enfoque no procedimento estatístico de definição de grupamentos municipais possibilitou visualizar as características demográficas e econômicas conjuntas desses municípios.

Destarte, para a análise multidimensional da pobreza, o estudo da SEI (2014) desenvolveu indicadores sintéticos com capacidade de agregar satisfatoriamente diversas variáveis importantes para o estudo da pobreza. A análise fatorial figurou como opção metodológica de construção dos indicadores, pois possibilita a redução do número de variáveis utilizadas e representar a estrutura de relação existente entre as mesmas. Para compor a análise o trabalho selecionou as dimensões demografia, saúde, moradia e renda. Em cada dimensão figura um conjunto de variáveis que buscam captar aspectos relacionados às condições de vida da população, as características de pobreza e seus domicílios. A princípio foram selecionadas seiscentas variáveis a partir do censo de 
2010, as quais foram tratadas e, por meio da construção de cada fator, identificaram-se as variáveis mais expressivas para a composição de cada dimensão (SEI, 2014).

Para a avaliação dos resultados, estabeleceram-se cinco grupos de pobreza denominados A, B, C, D e E. A média de referência é a magnitude 50 (cinquenta) para os valores dos centroides de agrupamentos. Assim, quanto mais abaixo da média forem os centroides de agrupamento, a condição de pobreza será considerada menos crítica para o grupo de municípios. Contrariamente, a situação será mais severa.

Portanto, boa parte dos trabalhos que tratam da análise da pobreza no estado da Bahia parte do princípio de que a insuficiência de renda é um parâmetro importante para a investigação do fenômeno, mas que há uma necessidade de se ampliar a perspectiva desta abordagem. Nesse sentido, coadunando com os programas de pesquisa desenvolvidos nas últimas décadas nacional e internacionalmente, preconiza-se o uso de outras variáveis não monetárias para retratar melhor a realidade da pobreza e, diante disso, se estabelecer políticas específicas para o seu enfrentamento. Entretanto, observam-se poucos estudos direcionados à análise multidimensional da pobreza para a Bahia. Nesse sentido, torna-se pertinente o estudo aqui desenvolvido, visando a contribuir com o debate e oferecer proposições de enfrentamento da pobreza de maneira a mitigar esse fenômeno no estado.

\section{METODOLOGIA}

\subsection{BASE DE DADOS E ESTRUTURA DAS DIMENSÕES}

A Pesquisa Nacional por Amostra de Domicílios (PNAD) foi utilizada para a construção dos indicadores multidimensionais de pobreza para este trabalho. Os períodos selecionados são os anos de 2006, 2007, 2008, 2009, 2011, 2012 e 2013. As dimensões e suas respectivas variáveis encontram-se detalhadas na Tabela 1 . São seis dimensões e vinte e duas variáveis derivadas a partir das variáveis originais extraídas das PNAD. A escolha das dimensões e variáveis seguiu o referencial teórico supracitado, tanto sob o enfoque das necessidades básicas quanto das capacidades.

Os indicadores estão definidos como $X_{i, k}^{l}$, sendo $i$ o número de pessoas que variam de 1 a $n$, $j$ o número de domicílios que variam de 1 a $h$ e $k$ as dimensões que variam de 1 a $m$. Os indicadores apresentam o valor máximo de 1 , situação de sem privação, e um valor mínimo de 0 , situação de privação total. A definição da variação dos indicadores entre 0 e 1 visa a reduzir os problemas de descontinuidade, mas é limitada pela informação disponível. A agregação das dimensões para a formação de cada indicador obedece à função $X_{i, k}=g_{k}\left(X_{i, k}^{l}, \ldots, X_{i, k}^{p}\right)$ para as variáveis $l=\{1, \ldots, p\} ; g_{k}($.$) uma função$ 
Tabela 1 - Dimensões e indicadores da pobreza multidimensional

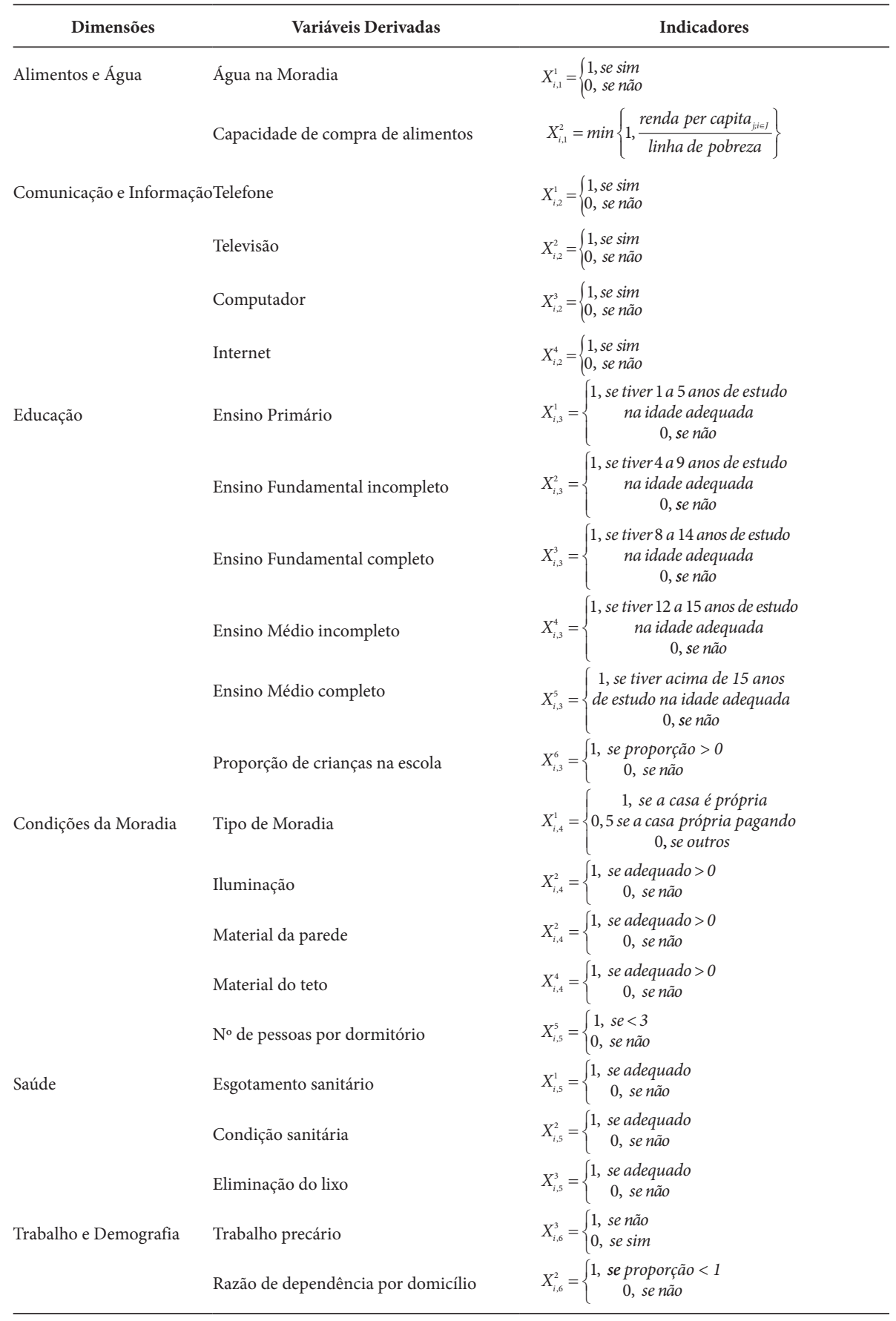

Fonte: Elaboração própria com base em dados da PNAD/IBGE. 
especifica para cada dimensão $k$. Por sua vez, define-se o nível de privação como $\hat{X}_{i, k}=$ $1-X_{i, k}$. Isso implica que há um gap relativo entre o nível individual $X_{\mathrm{i}, \mathrm{k}}$ e o limiar da privação $z=1$. A privação total ocorre no valor máximo de 1 (um) e o status sem privação no valor mínimo de 0 (zero).

\section{Quadro 1 - Descrição das dimensões}

\begin{tabular}{|c|c|c|}
\hline Dimensão & Descrição & Observação \\
\hline Alimentos e água & $\begin{array}{l}\text { Alimentação: captura a capacidade de } \\
\text { compra de alimentos; Água: mede se há } \\
\text { abastecimento de água apropriada na } \\
\text { moradia. }\end{array}$ & $\begin{array}{l}\text { Capacidade de compra relaciona renda per } \\
\text { capita do indivíduo com a linha de pobreza } \\
\text { utilizada. }\end{array}$ \\
\hline $\begin{array}{l}\text { Comunicação e } \\
\text { informação }\end{array}$ & $\begin{array}{l}\text { Indivíduos privados: não possuem meios } \\
\text { de informação para a vivência na } \\
\text { sociedade atual. }\end{array}$ & $\begin{array}{l}\text { As variáveis adotadas no estudo são: } \\
\text { telefone, televisão, computador e internet. }\end{array}$ \\
\hline Educação & $\begin{array}{l}\text { Sem privação: nível de escolaridade maior } \\
\text { do que o requerido para a idade; com } \\
\text { privação: indivíduos de } 18 \text { anos ou mais } \\
\text { sem o ensino médio concluído }\end{array}$ & $\begin{array}{l}\text { Lei de Diretrizes e Bases da Educação (Lei } \\
\text { 9.394/1996) afirma que há um nível de } \\
\text { escolaridade mínima requerida para uma } \\
\text { determinada faixa etária. }\end{array}$ \\
\hline Condições de Moradia & $\begin{array}{l}\text { Ausência de condições adequadas de } \\
\text { moradia com privação }\end{array}$ & $\begin{array}{l}\text { Variáveis: tipo de moradia, iluminação, } \\
\text { material de parede, material do teto, e } \mathrm{n}^{\circ} \\
\text { de pessoas por dormitório. }\end{array}$ \\
\hline Saúde & $\begin{array}{l}\text { Falta de acesso/acesso inapropriado a } \\
\text { qualquer uma das variáveis esgotamento } \\
\text { sanitário, condição sanitária e eliminação } \\
\text { do lixo }\end{array}$ & $\begin{array}{l}\text { Utilização de proxies por falta de variáveis } \\
\text { específicas disponíveis. A saúde é afetada } \\
\text { por essas variáveis. }\end{array}$ \\
\hline Trabalho e demografia & $\begin{array}{l}\text { Trabalho precário: trabalhador não } \\
\text { segurado pela previdência social e que não } \\
\text { contribua com nenhum outro instituto de } \\
\text { previdência; razão de dependência (RD) }\end{array}$ & $\begin{array}{l}\text { RD: trata da relação entre pessoas em } \\
\text { idade potencialmente inativa e pessoas em } \\
\text { idade potencialmente ativa; recorte: idade } \\
\text { inferior a } 14 \text { anos ou igual ou superior a } 60 \\
\text { anos foram definidas como dependentes; } \\
\text { Estatuto do Idoso (Lei } 10.741 / 2003 \text { ) }\end{array}$ \\
\hline
\end{tabular}

Fonte: Elaboração própria.

Salienta-se que as linhas de pobreza utilizadas para travar relação entre a capacidade de aquisição de alimentos e a renda per capita do indivíduo foram as definidas pelo Instituto de Estudo do Trabalho e Sociedade (IETS, 2014), elaboradas com base na Pesquisa de Orçamento Familiar (POF). Definidas as dimensões, variáveis e as formulações básicas de cada indicado, a próxima seção abordará a metodologia de cálculo utilizada.

\subsection{O MÉTODO DE BOURGUIGNON E CHAKRAVARTY}

Os procedimentos adotados para medir a pobreza influenciam na forma de entendê-la, analisá-la e, consequentemente, impactam nas decisões sobre políticas de enfrenta- 
mento do fenômeno. Dentre as diversas definições de pobreza e formas de mensuração, considerar as possibilidades de ser pobre em qualquer dimensão da pobreza apresenta-se como a forma mais simples desse procedimento. Isso implica na possibilidade de agregação de vários atributos de um mesmo indivíduo em um índice cardinal simples. Assim, de acordo com Bourguignon e Chakravarty (2003), dentro da abordagem multidimensional, a pobreza é definida considerando um vetor de características particulares. Dessa forma, a partir desse processo define-se o indicador de pobreza, para então classificar o indivíduo como pobre ou não pobre.

Para o desenvolvimento do indicador multidimensional de pobreza, denota-se, aqui, a função mj $P(X, z): M \times z \rightarrow R_{+}^{1}$ como a forma geral de sua apresentação. Nessa função, $X \in M$ é uma matriz de atributos, de dimensões $(n \times m)$, para $i=\{1,2, \ldots . m\}$ pessoas e $k=\{1,2, \ldots . m\}$ dimensões. Os tipos de atributos variam conforme o referencial do pesquisador, podendo considerar renda, educação, saúde entre outros. Por sua vez, para diferentes atributos selecionados, usa-se um vetor de limites ou "níveis minimamente aceitáveis", $z \in Z$.

Seguindo o método adotado por Bourguignon e Chakravarty (2003), o indicador multidimensional de pobreza é definido como

$$
P(X, z)=\frac{1}{n} \sum_{i=1}^{n} f\left(\begin{array}{l}
\max \left\{0 ;\left(1-\frac{x_{i, 1}}{z_{1}}\right)\right\} \\
, \ldots, \max \left\{0 ;\left(1-\frac{x_{i, k}}{z_{k}}\right)\right\}
\end{array}\right)
$$

$\mathrm{Na}$ forma geral, tem-se

$$
P(X, z)=\frac{1}{n} \sum_{i=1}^{n} f\left(X_{\hat{\imath}, 1, \ldots, X_{\hat{\imath}, m}}\right)
$$

O passo seguinte é definir $f(\cdot)$ na forma geral. Considerando a abordagem vinculativa e uma variação no índice de Foster et al. (1984) para capturar a intensidade da pobreza, denota-se a medida multidimensional de pobreza adotada nesse trabalho como:

$$
P(X, z)=\frac{1}{n} \sum_{i=1}^{n} f\left[{ }_{m}^{1} \sum_{i=1}^{n} X_{\hat{\imath}, k^{2}}\right]
$$

Parte-se do suposto de que as dimensões não são substituíveis, mas se inter-relacionam com o nível geral de pobreza na equação (2). Essa consideração é consistente com uma abordagem baseada em dimensões de bem-estar. Além disso, a repartição dos pesos foi hierarquizada para o nível individual para que o indicador seja sensível à distribuição de pobreza. Dessa maneira, dá-se maior peso às dimensões que apresentam um maior gap de privação e, em seguida, maior peso às pessoas com maiores ní- 
veis de privação. O nível individual de pobreza é definido como $P_{i} \frac{1}{m} \sum_{i=1}^{n} X \hat{\imath}, k^{2}$, onde a pobreza total é verificada quando o valor máximo é 1 (um) e a condição sem pobreza é identificada quando o valor mínimo de 0 é atingido.

A metodologia adotada estima o índice de incidência de cada dimensão (proporção de pobres) e os níveis de privação para diferentes regiões e grupos demográficos. Esse procedimento possibilita verificar a pobreza de forma mais qualitativa. Pode-se, assim, verificar se a incidência de pobreza ocorre mais na zona rural ou na zona urbana e em qual faixa etária ela é mais intensa. São perspectivas importantes para qualquer formulação de políticas de enfrentamento do problema por especificar as condições de pobreza por grupo. Dessa forma, em termos de cálculo do índice de incidência, considera-se que todas as pessoas que estão abaixo do limite em pelo menos uma variável sofrem privação (enfoque de união). A formalização é dada da seguinte maneira:

$$
\text { Sofre Privação }=\left\{\begin{array}{l}
\text { Sim; se } X_{\hat{\imath}, k}>0 \\
\text { Não; se } X_{\hat{\imath}, k}=0
\end{array}\right.
$$

O nível de privação para cada indivíduo em cada indicador é medido diretamente por $X_{i, k}^{\hat{l}}$. Mas o nível de privação individual em cada dimensão é determinado pelo processo de agregação correspondente a uma função $g_{k}($.). Dessa forma, os indicadores são agregados em nível para cada dimensão obedecendo à relação definida em $X_{i, k}=g_{k}\left(X_{i, k}^{l}, \ldots, X_{i, k}^{p}\right), \operatorname{com} l=\{1, \ldots, p\}$ A função $g_{k}($.$) é específica de cada dimensão$ $k$. Todos os indicadores têm o valor máximo de 1 (nível alcançado) e um valor mínimo de 0 (privação total), sendo essa definição utilizada para reduzir os problemas de descontinuidade. A definição de é dada pela equação (4).

$$
X_{i, k}=\frac{1}{p} \sum_{l=1}^{p} X_{i, k}^{l}
$$

Considerando todos os pontos supracitados, pode-se, a partir desse momento, estabelecer o nível de privação global. A decomposição para cada dimensão é denotada por:

$$
X_{\hat{k}}=\frac{1}{n} \sum_{i=1}^{n} X_{\hat{\imath}, k}
$$

Portanto, utiliza-se a equação (4) para mensurar o nível de privação global. Assim, computa-se $\left(X_{\hat{\imath}, k}\right)$ para cada dimensão e se há determinado nível de privação médio entre as variáveis. Por sua vez, utiliza-se $X_{\hat{\imath}, k}=1-X_{i, k}$ para identificar o nível de privação de cada dimensão. Conceitualmente, tem-se que o nível de privação, $X_{\hat{\imath}, k}$ é a diferença relativa entre o nível individual de $X_{i, k}$ e o limite da privação $Z_{k}=1$. A privação 
total ocorre quando o resultado é 1 (valor máximo) e a não privação ocorre quando o valor encontrado é 0 (valor mínimo).

Com base na equação (5), decompõe-se o nível de privação por região e grupo demográfico conforme equação (6), em que $S$ é o conjunto de grupos $\{1, \ldots, q\}$ e $\{1, \ldots, q\}, e X_{\hat{k}^{(s)}}=\frac{1}{n_{s}} \sum_{i_{s}=1}^{n_{s}} X_{i_{s}, \dot{\hat{k}}}$.

$$
X_{\hat{k}}=\sum_{s=1}^{q} \frac{n_{s}}{n} X_{\hat{\imath}, k^{(s)}}
$$

Portanto, utilizando os procedimentos acima descritos, têm-se os mecanismos para medir a pobreza na perspectiva multidimensional. Pode-se, a partir da seleção de dimensões e variáveis construídas e retiradas a partir dos microdados da PNAD, definir se um indivíduo se encontra ou não em condições de pobreza. Além disso, pode-se observar essa condição por nível de intensidade, por condição demográfica e região. Por outro lado, cabe ressaltar que a perspectiva conceitual adotada para definir pobreza debruça-se na abordagem das privações e das capacidades. Dessa maneira, a seção seguinte traz a análise das condições de pobreza multidimensional no estado da Bahia e, em especial, em seu meio rural, com base nessa perspectiva teórica e metodológica.

\section{RESULTADOS}

\subsection{INCIDÊNCIA DE PRIVAÇÃO E SUAS DIMENSÕES}

Ao analisar a pobreza como privação de fins, é possível considerar duas frentes mais importantes, ou seja, a que trata das necessidades básicas e outra referente às capacidades. Na primeira, o grau de pobreza é identificado pelos indicadores de acesso observados na Tabela 2. Por outro lado, a segunda consideração com base em Sen (1985) admite a importância da heterogeneidade dos indivíduos.

Considerando a dimensão água e alimentos, observa-se significativa evolução deste indicador no estado da Bahia. No ano de 2013 a porcentagem de domicílios não atendidos pelo abastecimento de água potável era de $9,81 \%$ contra $16,34 \%$ no ano de 2006. Por outro lado, quando se considera a capacidade de compra de alimentos, esta se refere à privação monetária. Nesse aspecto, de acordo com Silva et al. (2014), os domicílios são considerados privados se a renda per capita for inferior à linha pobreza, o que restringe a capacidade de consumo e, consequentemente, o suprimento das necessidades. Assim, a incidência de privação monetária ou capacidade de adquirir alimentos caiu de $14,57 \%$ para um nível de $6,47 \%$ no mesmo período. 
No que tange à dimensão de comunicação e informação estruturada conforme o acesso a telefone, televisão, computador e internet. Entre os anos de 2006 e 2013, todas as variáveis sofreram retração quanto à privação, o que contribuiu para a redução da pobreza nessa dimensão. É possível destacar os itens telefone e televisão como os que apresentam as maiores quedas relativas nos índices de privação no período, se comparados às variáveis computador e acesso à internet. Em 2006, os índices de privação de telefone e televisão eram respectivamente de $43,74 \%$ e $11,65 \%$. Por outro lado, no ano de 2013 atingiram níveis de 9,89 e 3,68\%. Entretanto, quando se trata da privação de computadores e internet, embora os graus de ambas tenham apresentado queda no período, ainda assim, permanecem consideravelmente elevadas. No ano de 2006, o nível de privação era de $88,09 \%$ para computadores e de $91,66 \%$ para internet e, em 2013 , respectivamente, de $61,89 \%$ e $67,08 \%$. Portanto, é possível que a redução da privação esteja correlacionada à difusão tecnológica ocorrida nos últimos anos, que proporcionou uma maior facilidade ao acesso às novas tecnologias de comunicação.

Tabela 2 - Incidência de privação no estado da Bahia, 2006-2013 (\%)

\begin{tabular}{|c|c|c|c|c|c|c|c|}
\hline Dimensões/Variáveis & 2006 & 2007 & 2008 & 2009 & 2011 & 2012 & 2013 \\
\hline Dimensão 1: Água e alimentos & 27,44 & 23,61 & 21,45 & 19,64 & 17,25 & 15,27 & 15,35 \\
\hline Água na Moradia & 16,34 & 13,07 & 12,65 & 11,50 & 9,95 & 9,86 & 9,81 \\
\hline Capacidade de compra de alimentos & 14,57 & 13,09 & 10,88 & 9,99 & 8,61 & 6,70 & 6,47 \\
\hline Dimensão 2: Comunicação e informação & 91,81 & 88,90 & 85,19 & 81,72 & 72,81 & 69,86 & 67,41 \\
\hline Telefone & 43,74 & 37,39 & 30,08 & 26,64 & 12,95 & 11,47 & 9,89 \\
\hline Televisão & 11,65 & 8,42 & 6,95 & 5,63 & 3,95 & 3,80 & 3,68 \\
\hline Computador & 88,09 & 84,64 & 80,78 & 76,89 & 67,96 & 64,89 & 61,89 \\
\hline Internet & 91,66 & 88,75 & 85,00 & 81,36 & 72,50 & 69,46 & 67,08 \\
\hline Dimensão 3: Educação & 96,96 & 96,76 & 96,95 & 95,88 & 95,29 & 95,20 & 94,49 \\
\hline Ensino Primário & 85,06 & 85,18 & 85,27 & 84,71 & 84,27 & 84,66 & 84,19 \\
\hline Ensino Fundamental incompleto & 94,77 & 94,73 & 94,36 & 93,78 & 93,33 & 93,13 & 92,48 \\
\hline Ensino Fundamental completo & 96,09 & 95,90 & 95,58 & 94,98 & 94,29 & 94,28 & 93,53 \\
\hline Ensino Médio incompleto & 96,88 & 96,65 & 96,40 & 95,77 & 95,11 & 95,05 & 94,35 \\
\hline Ensino Médio completo & 96,96 & 96,76 & 96,45 & 95,87 & 95,28 & 95,20 & 94,49 \\
\hline Proporção de crianças na escola & 4,00 & 4,24 & 2,46 & 2,72 & 2,06 & 1,59 & 1,82 \\
\hline Dimensão 4: Condições da moradia & 31,31 & 29,00 & 28,34 & 29,11 & 27,11 & 25,92 & 26,96 \\
\hline Tipo de moradia & 20,90 & 20,47 & 20,08 & 20,99 & 20,46 & 20,36 & 21,53 \\
\hline Iluminação & 3,00 & 2,16 & 1,99 & 1,58 & 1,25 & 0,94 & 0,69 \\
\hline Material da parede & 2,08 & 1,94 & 1,86 & 2,10 & 1,66 & 1,36 & 1,56 \\
\hline Material do teto & 0,81 & 0,59 & 0,92 & 1,01 & 0,85 & 0,50 & 1,09 \\
\hline No de pessoas por dormitório & 8,67 & 7,67 & 7,04 & 7,40 & 5,76 & 4,82 & 4,24 \\
\hline Dimensão 5: Saúde & 53,02 & 52,18 & 50,78 & 52,00 & 49,58 & 47,36 & 47,08 \\
\hline Esgotamento sanitário & 51,65 & 51,36 & 49,88 & 51,44 & 48,73 & 46,36 & 46,38 \\
\hline Condição sanitária & 20,56 & 20,57 & 19,56 & 18,98 & 17,37 & 19,79 & 18,00 \\
\hline Eliminação do lixo & 1,18 & 0,98 & 1,07 & 0,78 & 0,47 & 0,34 & 0,59 \\
\hline Dimensão 6: Trabalho e demografia & 72,49 & 73,04 & 72,61 & 71,62 & 69,37 & 69,34 & 69,41 \\
\hline Trabalho precário & 67,37 & 67,75 & 67,61 & 65,87 & 64,42 & 64,16 & 64,54 \\
\hline Razão de dependência por domicílio & 18,45 & 18,74 & 17,59 & 18,50 & 17,95 & 18,48 & 18,42 \\
\hline
\end{tabular}

Fonte: Elaboração própria com base em dados da PNAD/IBGE. 
Ainda com relação à Tabela 2, a dimensão educação é correspondente aos níveis educacionais estratificados, desde o ensino primário até o ensino médio completo. Ademais, a análise incorpora a proporção de crianças na escola referente ao total em cada domicílio. Nesse sentido, essa proporção diminui de forma acentuada entre 2006 e 2013, quando o grau de privação passou de $4,0 \%$ para $1,82 \%$, refletindo o processo de universalização do acesso ao ensino básico. De uma forma geral, a retração da privação educacional em todos os níveis de ensino foi muito pequena, passando de 96,96\% em 2006 para 94,49\% no ano de 2013. Mesmo assim, entre os níveis de escolarização destaca-se a queda da privação do ensino fundamental completo de 96,09\% para 93,53\% nos anos analisados.

A dimensão condições de moradia engloba os seguintes indicadores: tipo de moradia, iluminação, condição sanitária e material do teto. Entre os anos 2006 e 2013 observa-se considerável queda do índice de privação de $31,31 \%$ para $26,96 \%$. Os indicadores de privação de iluminação, qualidade da parede e material do teto acompanham essa tendência. Por outro lado, em 2006 a população baiana que vivia em domicílios com mais de três pessoas por quarto era de $8,67 \%$ contra $4,42 \%$ no ano de 2013.

Dentro das dimensões consideradas na análise, um aspecto relevante do ponto de vista social se refere às questões relacionadas à saúde, que geralmente estão associadas às condições de saneamento básico, cujo acesso apresenta forte correlação com os níveis de bem- estar da população. Neste item da pesquisa utilizou-se como variável proxy da dimensão saúde as condições sanitárias e de esgotamento, além da forma de eliminação do lixo. Espera-se que na medida em que tais condicionantes sejam adequados e apresentem um menor grau de privação, menor deverá ser a probabilidade da manifestação de doenças. Como observado, todos os indicadores apresentaram queda ao longo dos anos e a redução da privação da dimensão saúde que em 2006 era $53,02 \%$ passou a $47,08 \%$ no ano de 2013.

Por fim, a dimensão trabalho e demografia reflete respectivamente as condições de trabalho associadas a sua precariedade (trabalhador não segurado por instituto de previdência) e a proporção de pessoas dependentes por domicílio, menores de 14 e maiores de 60 anos de idade. Ambos indicadores de privação apresentam queda ao longo do tempo, embora se observe instabilidade do índice quanto à proporção de pessoas dependentes por domicílio, que em 2006 era de 18,45 \%, passando a 18,42\% no ano de 2013. Quanto às condições de trabalho no período, a privação destas caiu de 67,37\% para $64,54 \% \%$, portanto, um índice ainda bastante considerável em comparação com os padrões do mercado de trabalho em nível internacional. Entretanto, de forma geral, a dimensão trabalho e demografia indicam avanços na medida em que sua privação retrocede de 72,49\% para 69,41\% entre 2006 e 2013. 


\subsection{O COMPORTAMENTO DOS GAPS DE PRIVAÇÃO}

Como discutido na seção que trata da metodologia, os gap, ou lacunas de privação, representam a distância entre os indivíduos pobres e um determinado limite de pobreza total, variando entre 0 e 1 e calculados para cada dimensão. Tal indicador será analisado considerando o gênero e o espaço geográfico, levando em conta a região metropolitana de Salvador e os espaços metropolitano, urbano e rural do estado da Bahia entre os anos de 2006 e 2013.

Assim, levando em consideração o espaço geográfico em todas as dimensões, observa-se um predomínio dos níveis de privação de forma mais acentuada no meio rural baiano comparativamente às áreas urbanas e à região metropolitana, conforme aponta a Tabela 3. Corroborando esse processo, Lacerda e Neder (2010) mostram que entre os anos de 1995 e 2006 o gap médio da pobreza no estado da Bahia diminuiu de forma significativa. Entretanto, foi exatamente no meio rural que se identificou o gap médio mais elevado, o que indica um grau de pobreza mais intenso. Esse mesmo sentido é evidenciado no trabalho de Silva et al. (2014) para o Brasil, em que os gap de privação são mais acentuados em áreas rurais, comparativamente ao espaço urbano e metropolitano.

O primeiro gap de privação analisado se refere à dimensão água e alimentação, em que as evidências sinalizam a maior discrepância, relevando todas as dimensões consideradas de privação entre as zonas urbana, metropolitana e rural. O indicador deixa claro que possivelmente os níveis de insuficiência alimentar e sua correlação com a desnutrição são mais graves nas áreas rurais do estado da Bahia. Conforme a Tabela 3, a lacuna de privação nessas áreas era de 26,25\% em 2006, retraindo-se para 15,62\% em 2013. Embora se observem avanços no indicador, a lacuna de privação ao acesso à água e a alimentos ainda permanece em níveis consideravelmente elevados e com expressiva diferença em relação às áreas urbanas e metropolitana. Por outro lado, quando se incorpora à análise apenas o gênero ( masculino e feminino), as evidências apontam uma pequena variação entre os indivíduos ao longo do tempo. Assim, o gap agregado para a Bahia apresentou uma variação de $4,85 \%$ no período, o que, de uma forma geral, explicita uma melhoria ao acesso à água e à alimentação em todo o Estado.

No quesito comunicação e informação, o meio rural apresentou um gap de privação muito superior em comparação aos meios urbano e rural. Tal processo aponta o quanto ainda é deficiente o acesso aos meios globais de comunicação nos quais se incluem televisão, telefonia, computadores e Internet. De acordo com a Tabela 3, a área urbana foi o espaço geográfico em que foram mais evidentes os maiores avanços proporcionais. A lacuna de privação diminuiu de 43,71\% para 22,11\% entre 2006 e 2013. Ademais, nesse mesmo período, na região metropolitana a retração foi de 43,71\% para 
$22,11 \%$, e no campo foi de $76,82 \%$ para $55,41 \%$. Quando se consideram os grupos populacionais, os homens apresentaram maior lacuna de privação em relação às mulheres, de forma que o indicador para ambos diminuiu no período considerado.

Tabela 3 - Bahia: Gap da privação por dimensão, 2006 e 2013 (\%)

\begin{tabular}{lcccccc}
\hline \multirow{2}{*}{ Região/Grupo } & \multicolumn{2}{c}{ Água e alimentos } & \multicolumn{2}{c}{ Comunicação e informação } & \multicolumn{2}{c}{ Educação } \\
\cline { 2 - 7 } & $\mathbf{2 0 0 6}$ & $\mathbf{2 0 1 3}$ & $\mathbf{2 0 0 6}$ & $\mathbf{2 0 1 3}$ & $\mathbf{2 0 0 6}$ & $\mathbf{2 0 1 3}$ \\
\hline Bahia & 11,60 & 6,75 & 58,78 & 35,63 & 86,54 & 85,09 \\
\hline Metropolitano & 5,18 & 3,04 & 43,71 & 22,11 & 83,88 & 81,48 \\
Urbano & 8,17 & 4,79 & 58,63 & 34,01 & 86,78 & 85,37 \\
Rural & 26,25 & 15,62 & 76,82 & 55,41 & 89,17 & 88,76 \\
Homens & 11,61 & 6,89 & 59,58 & 36,46 & 87,05 & 86,21 \\
Mulheres & 11,58 & 6,63 & 58,02 & 34,86 & 86,05 & 84,06 \\
\hline \multirow{2}{*}{ Região/Grupo } & Condições da moradia & \multicolumn{2}{c}{ Saúde } & Trabalho e demografia \\
\cline { 2 - 7 } & $\mathbf{2 0 0 6}$ & $\mathbf{2 0 1 3}$ & $\mathbf{2 0 0 6}$ & $\mathbf{2 0 1 3}$ & $\mathbf{2 0 0 6}$ & $\mathbf{2 0 1 3}$ \\
\hline Bahia & 6,93 & 5,56 & 24,46 & 21,65 & 42,91 & 41,47 \\
\hline Metropolitano & 6,64 & 5,80 & 6,84 & 4,91 & 36,98 & 33,34 \\
Urbano & 6,70 & 6,09 & 18,47 & 15,03 & 46,77 & 44,81 \\
Rural & 7,75 & 4,08 & 57,57 & 56,60 & 41,86 & 43,60 \\
Homens & 7,06 & 5,60 & 25,15 & 22,53 & 42,49 & 40,79 \\
Mulheres & 6,81 & 5,53 & 23,79 & 20,84 & 43,31 & 42,11 \\
\hline
\end{tabular}

Fonte: Elaboração própria com base em dados da PNAD/IBGE.

No tocante à dimensão educação, o gap de privação sinalizou um tímido avanço com pequena variação de $1,45 \%$ entre 2006 e 2013. Contudo, é exatamente no meio rural que se observa o maior gap com tímida mudança de $89,17 \%$ para $88,76 \%$. Entretanto, a maior variação ocorreu na área metropolitana, quando o gap de privação no ano de 2013 alcançou 81,48\%. Considerando homens e mulheres, o comportamento do gap de privação nessa dimensão seguiu trajetória similar aos evidenciados nos espaços geográficos, ou seja, variando muito pouco, mas ao mesmo tempo indicando avanços e redução da privação dos indivíduos em relação à educação. Nesse mesmo sentido, Lacerda e Neder (2010) observam que apesar dos avanços no grau de instrução educacional da população baiana, em decorrência da expansão dos anos de escolaridade no período recente, vê-se claramente que a magnitude desse processo ainda é bastante insignificante e tem ocorrido de forma muita lenta ao longo dos anos. Diante desse cenário de adversidade, políticas de acesso à educação de qualidade, em especial nas áreas rurais, são de fundamental importância para a redução das desigualdades de educação e renda e, consequentemente, dos níveis de pobreza.

Quanto aos gap relacionados às dimensões de condições de moradia, saúde, trabalho e demografia, estes corroboram a análise anterior correspondente aos indicadores de privação. Conforme a Tabela 2, em todos eles a variação foi muito pequena entre os anos de 2006 e 2013. Em relação às condições de moradia, a lacuna de privação na 
zona rural passou de 7,75\% em 2006 para um nível de 7,18\% em 2013. Nesse mesmo período, o gap de privação diminuiu mais fortemente na área metropolitana do estado de um nível de 6,64\% para 5,80\%. Analisando esse processo por gênero, pode-se destacar que os indivíduos do gênero masculino apresentaram os maiores gap de privação, com um valor de 5,60\% no ano de 2013.

Dentro da dimensão saúde, observam-se avanços, com redução da lacuna de privação de um patamar de 24,24\% em 2006 para 21,65\% no ano de 2013, o que sinaliza uma melhoria nas condições de saúde da população no estado da Bahia. Entretanto, uma das características predominantes no Brasil é o fato de que as áreas urbanas apresentam maior acessibilidade aos meios de saúde comparativamente às áreas rurais. Assim, as evidências indicam um predomínio e forte dispersão do acesso a este meio nas cidades (zona urbana e região metropolitana) em detrimento da área rural. A lacuna de privação nas áreas urbanas caiu de $18,47 \%$ para $15,03 \%$ contra apenas $57,57 \%$ e $56,60 \%$ no meio rural baiano entre 2006 e 2013, o que indica necessidade de políticas e ajustes na resolução deste abismo no sentido de reduzir tais disparidades. No que tange ao gênero, os homens apresentaram uma maior privação da saúde frente às mulheres.

Destarte, considerando o gap de privação da dimensão trabalho e demografia, este explicitou leve recuo em todas as áreas. Entretanto, mais uma vez o meio rural se destacou por apresentar um indicador de menor variação entre 2006 e 2013, embora a lacuna de privação tenha se mostrado aquém da apontada no meio urbano. Neste último, o gap de privação recuou de $46,77 \%$ para $44,81 \%$, enquanto na zona rural o mesmo indicador seguiu a mesma tendência, passando de $41,86 \%$ para $40,60 \%$ no mesmo espaço temporal. Por outro lado, o gap de privação desta dimensão para as mulheres foi superior ao observado para os homens, o que possivelmente reflete a discriminação no mercado de trabalho entre os gêneros.

\subsection{ANÁLISES DA POBREZA MULTIDIMENSIONAL}

Conforme Lacerda e Neder (2010), o critério de avaliação do grau de pobreza pelo o enfoque multidimensional desmistifica a ideia de que o crescimento econômico e o nível de renda são condições necessárias e suficientes para a resolução dos entraves associados à maximização do bem estar social. A abordagem multidimensional, parte do princípio de que o grau de pobreza em regiões com menor dinamismo econômico e social não poderia estar restrita apenas a variável renda, mas a um conjunto de aspectos e características sociais mais abrangentes.

Nesse sentido, como evidenciado anteriormente, o comportamento das dimensões e gap de privação sugerem uma evolução em relação à redução da pobreza multidi- 
mensional entre os grupos e áreas geográficas no estado da Bahia entre 2006 e 2013. A Tabela 4 confirma o que foi apontado pela análise dos indicadores, sinalizando uma queda da pobreza multidimensional em todas as áreas geográficas e grupos que foram incluídos na pesquisa.

Conforme aponta a Tabela 4, a variação da pobreza multidimensional no estado da Bahia foi de aproximadamente $-4,41 \%$, dada a retração do índice de pobreza de $28,0 \%$ em 2006 para 23,59\% no ano de 2013. Tal comportamento reflete a dinâmica recente dos índices de pobreza em todo o Brasil e especificamente na região Nordeste. Segundo Silva et al. (2013), entre os anos de 2006 e 2012 a redução observada dos níveis de pobreza multidimensional foi de respectivamente 3,01\% e 3,60\%, para o Brasil e o Nordeste. Particularmente, pode-se destacar o Nordeste como uma das regiões em que a redução da pobreza foi mais significante no período, ficando atrás apenas da região Norte, que embora tenha apresentado a maior redução do nível de pobreza ainda permanece com a maior proporção de pobres no país.

Tabela 4 - Pobreza multidimensional por áreas e grupos na Bahia, 2006-2013 (\%)

\begin{tabular}{lllllllll}
\hline & \multicolumn{7}{c}{ Pobreza Multidimensional } & Variação \\
\cline { 2 - 6 } & $\mathbf{2 0 0 6}$ & $\mathbf{2 0 0 7}$ & $\mathbf{2 0 0 8}$ & $\mathbf{2 0 0 9}$ & $\mathbf{2 0 1 1}$ & $\mathbf{2 0 1 2}$ & $\mathbf{2 0 1 3}$ & \\
\hline Bahia & 28,00 & 27,13 & 26,25 & 25,64 & 24,08 & 23,93 & 23,59 & $-4,41$ \\
\hline Metropolitano & 21,85 & 21,21 & 20,58 & 20,38 & 19,41 & 19,00 & 18,66 & $-3,19$ \\
Urbano & 27,06 & 26,09 & 25,19 & 24,38 & 23,22 & 22,85 & 22,67 & $-4,39$ \\
Rural & 37,18 & 35,70 & 34,19 & 33,97 & 32,32 & 32,33 & 31,57 & $-5,61$ \\
\hline Homens & 28,24 & 27,36 & 26,45 & 25,92 & 24,38 & 24,24 & 23,86 & $-4,38$ \\
Mulheres & 27,77 & 26,92 & 26,06 & 25,37 & 23,81 & 23,65 & 23,35 & $-4,42$ \\
\hline Crianças & 27,76 & 26,71 & 25,48 & 24,82 & 23,30 & 22,53 & 22,29 & $-5,47$ \\
Adolescentes & 27,50 & 27,00 & 26,25 & 25,22 & 23,40 & 23,31 & 22,85 & $-4,65$ \\
Jovens & 28,07 & 26,93 & 25,94 & 25,53 & 23,72 & 23,72 & 23,38 & $-4,69$ \\
Adultos & 26,82 & 26,18 & 25,44 & 24,69 & 23,23 & 23,03 & 22,58 & $-4,24$ \\
Idosos & 35,60 & 34,50 & 33,95 & 33,59 & 31,17 & 32,79 & 32,50 & $-3,10$ \\
\hline Branca & 26,63 & 25,75 & 25,41 & 24,49 & 23,08 & 23,10 & 22,30 & $-4,33$ \\
Não Branca & 28,37 & 27,51 & 26,48 & 26,00 & 24,36 & 24,15 & 23,94 & $-4,46$ \\
\hline
\end{tabular}

Fonte: Elaboração própria com base em dados da PNAD/IBGE.

Entretanto, quando o foco da análise da pobreza multidimensional muda para o meio rural do estado da Bahia, observa-se que, historicamente, o nível de pobreza e extrema pobreza no Brasil e, sobretudo, no Nordeste, tem se concentrado principalmente nesse meio, tornando os indivíduos presos ao que se denomina "armadilha da pobreza”. De acordo com Rocha (2006), até então, aproximadamente metade da população rural nordestina era considerada pobre. A explicação para isto decorreria principalmente de dois fatores mais importantes. Em primeiro lugar, a ausência de uma fonte de renda permanente e a precariedade das ocupações com impactos diretos sobre o processo de insegurança alimentar. Em segundo lugar, o meio rural tem se caracteri- 
zado ao longo do tempo como aquele em que as deficiências relacionadas à aplicabilidade de políticas públicas e sociais têm dificultado o acesso da população à educação e à saúde de qualidade, com possíveis efeitos adversos sobre as demais dimensões.

Nesse sentido, avaliando a dinâmica da pobreza multidimensional nas áreas rurais da Bahia, evidencia-se uma queda de 5,61\%, portanto, superior aos níveis observados nas áreas urbana e metropolitana, cujas respectivas variações de pobreza foram de $-4,39 \%$ e $-3,19 \%$. Assim, no ano de 2006, o índice de pobreza no meio rural, que era de $37,18 \%$, retrocedeu para $31,57 \%$ no ano de 2013. Essa tendência sugere que a população residente nesse meio melhorou os níveis de bem- estar associado às multidimensões que refletem o grau de pobreza. Ademais, o direcionamento de uma série de políticas públicas ao campo, como as transferências de renda e a expansão do crédito, foi fundamental a esse processo. Por outro lado, mesmo com a melhoria das condições de vida, as disparidades ainda resistem entre as áreas, embora em menor magnitude, visto que a dinâmica de distribuição dos efeitos sobre a pobreza ocorreu de forma heterogênea no espaço.

Destarte, quando se incorpora os grupos à análise da pobreza multidimensional, considera-se a inclusão de homens e mulheres, crianças, jovens, adultos, idosos e indivíduos que se declararam na amostra serem da raça branca ou não. Como mostra a Tabela 4, o grau de pobreza diminuiu para todos os componentes que correspondem à fração grupo. Ao se tratar especificamente do componente gênero, o indicador de pobreza entre homens e mulheres explicita variações respectivamente de $-4,38 \%$ e $-4,42 \%$, sinalizando uma pequena diferença entre ambos quanto ao grau de pobreza.

\section{CONSIDERAÇÕES FINAIS}

O presente artigo buscou analisar a pobreza multidimensional no estado da Bahia entre os anos de 2006 e 2013, observando a dinâmica e a tendência desse processo entre os espaços geográficos e grupos considerados. Assim, espera-se que as evidências empíricas apontadas sejam um instrumento fundamental de auxílio à formulação e execução de políticas públicas de enfrentamento à pobreza.

Os resultados apontam evidências da queda nos indicadores de incidência de privação por dimensão, gap de privação e de pobreza multidimensional, explicitando avanços em relação à redução da pobreza em suas várias dimensões. Contudo, discrepâncias ainda são observadas nos espaços geográficos analisados e entre os grupos que foram incorporados à análise.

Quando se refere ao meio rural, observa-se retração dos indicadores de privação e maior acessibilidade dos indivíduos às dimensões consideradas no estudo, refletidos através dos gap de privação. Assim, pode-se destacar o índice de privação de água e 
alimentos, que diminuiu de forma significativa, indicando maior acessibilidade às condições mínimas de sobrevivência ligadas à alimentação. Ademais, as famílias no meio rural passaram a ter maior acesso aos meios de comunicação e informação, fundamentais à inserção e inclusão social. Ademais, em se tratando dos grupos, todos os indicadores apresentaram trajetória semelhante aos observados nos espaços geográficos, com uma explícita tendência de queda da privação com rebatimentos positivos sobre a pobreza multidimensional dos indivíduos.

Tratando-se especificamente do indicador de pobreza multidimensional, este apresentou expressiva retração para o estado da Bahia durante o período analisado. Seguindo a mesma tendência, o indicador para as áreas rurais apontou redução da proporção de pobres com uma variação superior às evidenciadas para as áreas urbanas. Mesmo assim, o abismo da pobreza em relação às duas áreas avaliadas ainda é bastante relevante e reflete em parte a deficiência de políticas públicas frente aos ajustes de correção do problema.

No que tange aos grupos, o indicador de pobreza multidimensional apresentou retração para todos eles. Quando se compara o nível de pobreza entre homens e mulheres, observa-se uma pequena diferença entre ambos, mas significativa variação ao longo do período. Por outro lado, no que tange às faixas etárias, foram as crianças que evidenciaram a mais significativa retração do indicador em relação à pobreza, o que indica um maior nível de bem-estar e melhoria nas condições de vida dos indivíduos nessa faixa de idade.

\section{REFERÊNCIAS}

ALKIRE, S.; FOSTER, J. Understanding and misunderstanding of Multidimensional Poverty measurement. Working paper, Oxford poverty \& Human Development Initiative, University of Oxford, n. 43, may 2011.

ALKIRE, S.; FOSTER, J. “Counting and multidimensional poverty”. In: VON BRAUN J. (Ed.) The poorest and hungry: assessment, analysis and actions. Washington D. C.: International Food Policy Research Institute, 2009.

ANAND, S. e A. SEN. Concepts of Human Development and Poverty: A Multidimensional Perspective. New York: Human Development Papers, United Nations Development Programme, 1997.

BARROS, R. P. et al. Pobreza multidimensional no Brasil. Texto para Discussão, IPEA, Rio de Janeiro, n. 1227, out. 2006. Disponível em: <http://www.ipea.gov.br/sites/000/2/publicacoes/tds/td_1227.pdf >. Acesso em: 23 mar. 2014.

BOURGUIGNON, F.; CHAKRAVART, S. The measurement of multidimensional poverty. The Journal of Economic Inequality, v. 1, p. 25-49, 2003. 
FOSTER, J. et al. A class of decomposable poverty measures. Econometrica, v. 52, n. 3, p. 761-766, mai. 1984.

IBGE - Instituto Brasileiro de Geografia e Estatística. Pesquisa Nacional por Amostra de Domicílios - PNAD. Rio de Janeiro: IBGE, 2010.

IETS - INSTITUTO DE ESTUDOS DO TRABALHOS E SOCIEDADE. Disponível em: <http:// www.iets.org.br/dado/pobreza-e-indigencia>. Acesso em: 10 mar. 2014.

LACERDA, F. C. C.; NEDER, H. D. Pobreza Multidimensional na Bahia: uma análise fundamentada no indicador multidimensional de pobreza. Revista Desenbahia, v. 7, p. 33-70, 2010.

KUKLYS, W. Amartya Sen's capability approach: theoretical insights and empirical applications. New York: Springer Berlin Heidelberg, 2005.

MIDEROS, A. M. Ecuador: definición y medición multidimensional de la pobreza, 2006-2010. Revista de la CEPAL, n. 108, p. 51-70, 2012.

ROCHA, S. Alguns Aspectos Relativos à Evolução 2003-2004 da Pobreza e da Indigência no Brasil. Rio de Janeiro: IETS, jan. 2006. Disponível em: <http://www.direito.usp.br/faculdade/eventos/evolucao_pobreza.pdf $>$. Acesso em: 23 mar. 2014.

SALAMA, P.; DESTREMAU, B. O tamanho da pobreza: economia política da distribuição de renda. Rio de Janeiro: Garamound, 1999.

SEI - SUPERINTENDENCIA DE ESTUDOS ECONÔMICOS E SOCIAIS DA BAHIA. Pobreza na Bahia em 2010: dimensões, territórios e dinâmicas regionais. Série estudos e pesquisas, $\mathrm{n}$. 97, Salvador: SEI, 2014.

SEN, A. K. Poverty: an ordinary approach to measurement. Econometrica, v. 44, p. 219-231, 1976.

SEN, A. K. Commodities and capability. New York: Oxford University Press, 1985.

SEN, A. K. "Capability and well-being". In: SEN, A.; NUSSBAUM, M. (Eds.) The quality of life. Oxford: Clarendon Press, 1993, p. 30-55.

SEN, A. K. Desenvolvimento como liberdades. São Paulo: Companhia das Letras, 2000.

SILVA et al. Análise da pobreza multidimensional no Brasil. In: ENCONTRO NACIONAL DE ECONOMIA, 42, Natal/RN, 9-12 dez. 2014.

SILVA, M. C. P.; BARROS, R. P. Pobreza multidimensional no Brasil. In: ENCONTRO NACIONAL DE ECONOMIA, 34, Salvador / BA, 5-8 dez. 2006.

SILVA, A. M. R.; NEDER, H. D. Abordagem das capacitações: um estudo empírico sobre pobreza multidimensional no Brasil. In: CONFERÊNCIA LATINO AMERICANA E CARIBENHA SOBRE ABORDAGEM DAS CAPACITAÇÕES E DESENVOLVIMENTO HUMANO, 3, Porto Alegre / RS, 2010.

THORBECKE, E. "Multidimensional poverty: conceptual and measurement issues". In: KAKWANI, N.; SILBER, J. (Eds.). Many dimensions of poverty. London, UK: Palgrave Macmillan, 2008, p. 3-19.

UNDP - UNITED NATIONS DEVELOPMENT PROGRAMME. "Poverty in the human development perspective: concept and measurement”. In: UNPD (Org.) Human Development Report 1997. Human Development to Eradicate Poverty. New York: Oxford University Press, 1997, p. 15-23. 